(C) 2011 IEEE. Personal use of this material is permitted. Permission from IEEE must be obtained for all other uses, in any current or future media, including reprinting/republishing this material for advertising or promotional purposes, creating new collective works, for resale or redistribution to servers or lists, or reuse of any copyrighted component of this work in other works. 


\section{High Power Amplifie Pre-Distorter Based on Neural-Fuzzy Systems for OFDM Signals}

Víctor P. Gil Jiménez, Member, IEEE, Younes Jabrane, Ana García Armada, Senior Member, IEEE, Brahim Ait Es Said, and Abdellah Ait Ouahman

\begin{abstract}
In this paper, a novel High Power Amplifier (HPA) pre-distorter based on Adaptive Networks - Fuzzy Inference Systems (ANFIS) for Orthogonal Frequency Division Multiplexing (OFDM) signals is proposed and analyzed. Models of Traveling Wave Tube Amplifiers (TWTA) and Solid State Power Amplifiers (SSPA), both memoryless and with memory, have been used for evaluation of the proposed technique. After training, the ANFIS linearizes the HPA response and thus, the obtained signal is extremely similar to the original. An average Error Vector Magnitude (EVM) of $10^{-6}$ can be easily obtained with our proposal. As a consequence, the Bit Error Rate (BER) degradation is negligible showing a better performance than what can be achieved with other methods available in the literature. Moreover, the complexity of the proposed scheme is reduced.
\end{abstract}

Index Terms - High power amplifiers, inferences techniques, linearization techniques, memory, memoryless.

\section{INTRODUCTION}

$\mathbf{O}$ RTHOGONAL Frequency Division Multiplexing (OFDM) is one of the main modulations used for broadband broadcasting systems such as Digital Video Broadcasting - Terrestrial (DVB-T) [1], Digital Audio Broadcasting (DAB) [2] or Digital Radio Mondiale (DRM) [3], as well as other broadband wireless systems such as IEEE 802.16 [4], [5], and it is a promising technique for future systems. The main reason for the choice of OFDM is its robustness against the multipath effects of wireless channels. However, OFDM suffers from high power envelope fluctuations Since information in OFDM is carried both in amplitude and phase, it requires employing a very linear High Power Amplifie (HPA) in order not to distort the signal, and therefore, the information being transmitted. In broadcasting systems, very efficien amplifier are used, usually of two types, namely, Traveling Wave Tube Amplifier (TWTA) or Solid State Power Amplifier (SSPA). Unfortunately, these amplifier are highly non-linear and thus, large back-offs are

This work was supported in part by projects MULTIADAPTIVE (TEC2008-06327-C03-02)

and AECI Program of Research Cooperation

with Morocco. needed in order not to distort the signal. As a consequence, the efficien y is considerably reduced.

In order to avoid the use of large back-offs, there exists an extensive amount of literature about techniques for reduction of the power envelope fluctuations Usually, these fluctuation are measured by the Peak to Average Power Ratio (PAPR) - also known as PAR or PAP — or recently, by the Cubic Metric (CM). Examples of these techniques are PTS (Partial Transmit Sequences) [6], [7], SLM (Selective Mapping) [8], [9], TR (Tone Reservation) [10], coding such as using Golay Sequences [11], [12] or Reed-Solomon codes [13], and interleaving [14], [15]. The reduction obtained with these techniques relies on the idea that different OFDM symbols exhibit different envelope fluc tuations. On the other hand, if some distortion is allowed, the envelope fluctuation can be reduced significantly In this group of distortion-based techniques can be found techniques such as AC (Amplitude Clipping) [16], ACF (Amplitude Clipping and Filtering) [17], TI (Tone Injection) [10], ACE (Active Constellation Expansion) [18] or similar approaches to ACE [19]. Besides, there are also companding methods such as in [20] for peak power reduction.

However, even with a signal with low power envelope fluctua tions, the non-linearity of HPA will introduce a signal distortion, and thus, the system performance will be compromised [21]. In order to overcome this issue, it is possible to pre-distort the signal before the HPA in such a way that, after passing through the HPA, the output signal will be similar to the originally transmitted one, i.e., to pre-compensate the distortion caused by the HPA, or to linearize the HPA. Several authors have proposed different architectures for such predistorters for OFDM signals [21]-[35]. All of them try to estimate and linearize the AM/AM (amplitude modulation / amplitude modulation) and AM/PM (amplitude modulation / phase modulation) characteristics of the HPA, usually done by large Look Up Tables (LUT). If the HPA has memory, other techniques can be applied such as [30] or [31], where a 2D LUT is proposed for the linearization. In order to avoid these LUTs, several schemes have been proposed.

The main contributions are based on polynomial approaches, which, basically, try to reduce the number of coefficient for the full Volterra nonlinearity [22]. In [36], a joint LUT-based and polynomial-based method is described with significan reduction in memory requirements. In [23]-[25], the inverse polynomial with reduced members is proposed for memoryless systems. Then, in [26], a Hammerstein identificatio system is used for improving polynomial performance in scenarios with memory. In [27], a learning structure is proposed based on a polynomial approximation to linearize the HPA. In [28], a triangular polynomial is used to simplify the Volterra's series. In [29], authors propose a structure of a HPA predistorter based on a piece-wise linear approximation of the SSPA inverse characteristics, neglecting the AM/PM conversion. Next, an 
adaptation algorithm is derived that requires a considerable number of iterations. In [35], the characteristic of the predistorter is derived based on the extension of Saleh's model for HPA and a simple linear-log model. Numerical results show that it is robust for Global Broadcasting Service (GBS) applications. Another way to tackle the problem is to jointly reduce the power envelope fluctuation and predistort the signal as in [21], [34], and [37]. In [34] the TR technique is used for reducing the PAPR before predistortion and thus, the constraints on the predistorter can be relaxed, whereas in [37], the SLM or PTS techniques are used to decrease the nonlinear distortion [30] or [31].

Since the nonlinearity of HPA can be learned, in [32], [33], [38]-[40] and [41], instead of a linearization, a learning process based on Neural Networks (NN) is proposed for the predistorter. The idea is to make the $\mathrm{NN}$ learn on how to distort the input signal. However, due to limitations of $\mathrm{NN}$ that we explain in Section III, the system is complex even after training, and there is a residual performance degradation. In order to overcome these problems, we propose to use the Adaptive Networks based on Fuzzy Inference Systems (ANFIS) technique [42]. ANFIS was also proposed for pre-distorting Wideband Code Division Multiple Access (WCDMA) signals [43], [44], although memory had not been taken into account and the proposal was only valid for low density modulation such as QPSK. In this paper, after learning the nonlinearities of the HPA (either memoryless or with memory), we design a predistorter based on ANFIS that linearizes the AM/AM and the AM/PM conversion of the HPA, recovering the originally transmitted signal with an Error Vector Magnitude (EVM) around $0.001 \% \mathrm{rms}$, and valid for high density modulations such as 64-QAM. Thus, the Bit Error Rate (BER) performance degradation is negligible. The ANFIS pre-distorter scheme here proposed is valid for any kind of amplifie, independently of its model that we solely use in order to test and validate the results. Besides, we provide the analytical calculation of the EVM based on the trained parameters, and we show that the obtained EVM is much better than what other previous methods in the literature provide with very low complexity once the ANFIS has been trained. In summary, our proposal obtains almost negligible performance loss with a low increase in system complexity for memoryless and memory HPA scenarios.

Notation: In this paper, the following notation will be used. Boldface symbols will be used for vectors while normal-face for scalars. Time-domain signals will be denoted with small-case letters, whereas frequency-domain signals will use capitalized letters. $\mathcal{R}\{x\}$ and $\mathcal{I}\{x\}$ account for the real and imaginary part of $x$, respectively. And, $|x|$ denotes the absolute value of $x$, whereas $\phi(x)$ is the phase of $x$.

The paper is organized as follows. First, the system model is presented and the effects of nonlinearity in terms of constellation wrapping, Symbol Error degradation and out of band radiation are discussed in Section II. Next, in Section III, the ANFIS system is proposed and analyzed, including the complexity analysis. After that, in Section IV, the EVM is introduced and theoretically evaluated in our scheme. Finally, in Sections V and $\mathrm{VI}$, the results are presented and the conclusions are drawn, respectively.

\section{SYSTEM MODEL}

In this section the OFDM signal and the amplifie models and effects are described.

OFDM Signal Model: In a multi-carrier system such as OFDM, the time-domain complex base-band transmitted signal $\mathrm{x}^{\ell}$ for the $\ell$-th symbol can be written as

$$
\mathbf{x}^{\ell}=x^{\ell}[n]=\frac{1}{\sqrt{N}} \sum_{k=0}^{N-1} S_{k}^{\ell} e^{\frac{j 2 \pi k n}{N}}, \quad \begin{aligned}
& n=0 \cdots N-1 \\
& \ell=0 \cdots \infty
\end{aligned}
$$

where $N$ is the number of sub-carriers and $S_{k}^{\ell}$ is the frequencydomain complex base-band symbol modulated on the $k$-th subcarrier (belonging for example to an M-QAM constellation) at OFDM symbol $\ell$. The $\ell$-th symbol can be also expressed as

$$
\mathbf{x}^{\ell}=\left|x^{\ell}[n]\right| e^{j \phi\left(x^{\ell}[n]\right)}
$$

Amplifier Model: There are mainly two types of nonlinear amplifie models, namely, the memoryless HPA and the HPA with memory. Examples of models for the memoryless HPA are Saleh [45], [46] and Rapp model [47] for the TWTA and SSPA, respectively. Whereas for the HPA with memory, the common models are Saleh [45], Wiener [48], the parallel Wiener [49] and the polynomial models [50].

If the HPA frequency response exhibits a similar behavior in the whole working range, the HPA can be considered memoryless. In this case, for the TWTA or SSPA, the output $y(t)$ of the nonlinear amplifie is given by

$$
y(t)=F(|x(t)|) e^{j[\varphi(|x(t)|)+j \phi(x(t))]}
$$

where $F($.$) is the AM/AM conversion function and \varphi($.$) is the$ $\mathrm{AM} / \mathrm{PM}$ conversion introduced by the amplifier It should be noted that both of them depend on the absolute value of the input signal $|x(t)|$. According to Saleh's model [45], the AM/AM conversion and the AM/PM conversion can be expressed as

$$
\begin{aligned}
& F(|x(t)|)=\frac{\alpha_{\|}|x(t)|}{\left(1+\beta_{||}|x(t)|^{2}\right)} \\
& \varphi(|x(t)|)=\frac{\alpha_{\varphi}|x(t)|^{2}}{\left(1+\beta_{\varphi}|x(t)|^{2}\right)}
\end{aligned}
$$

where $\alpha_{\|}$is the small signal gain, $A_{\text {sat }}=1 / \sqrt{\beta_{\|}}$is the input saturation voltage of TWTA and $A_{\max }=\left(\alpha_{\|} / 2 \sqrt{\beta_{\|}}\right)=$ $\left(\alpha_{\|} A_{\text {sat }} / 2\right)$ stands for the maximum output amplitude. Similar meaning have the parameters for the phase. Two different TWTA have been used with typical values for these parameters. The TWTA1 with $\alpha_{\|}=2, \beta_{\|}=1, \alpha_{\varphi}=4$ and $\beta_{\varphi}=9$; and TWTA2 with $\alpha_{\|}=3, \beta_{\|}=2, \alpha_{\varphi}=2$ and $\beta_{\varphi}=7$ [38].

For the SSPA, the modifie Rapp model [51] is usually utilized. In this model, the $\mathrm{AM} / \mathrm{AM}$ and $\mathrm{AM} / \mathrm{PM}$ conversion can be expressed as

$$
F^{\prime}(|x(t)|)=g \frac{|x(t)|}{\left(1+\left(\frac{g|x(t)|}{A_{\text {sat }}}\right)^{2 s}\right)^{\frac{1}{2 s}}}
$$




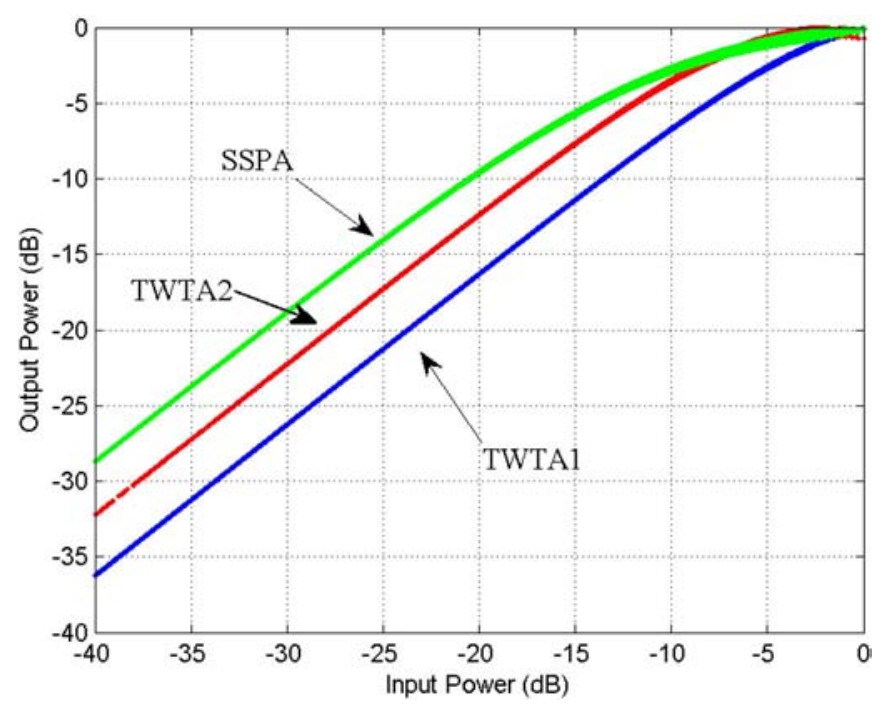

Fig. 1. HPA non-linear response for the modulo. $\mathrm{IBO}=2 \mathrm{~dB}$. TWTA $1=$ $\alpha_{\|}=2, \beta_{\|}=1$, TWTA2 $=\alpha_{\|}=3$, and $\beta_{\|}=2$. Memoryless HPA.

$$
\varphi^{\prime}(|x(t)|)=\frac{\alpha|x(t)|^{q_{1}}}{\left(1+\left(\frac{|x(t)|}{\beta}\right)^{q_{2}}\right)}
$$

where $g$ is the small gain signal, $s$ is a smoothness factor parameter, $A_{\text {sat }}$ is the saturation level with a similar meaning as in the TWTA, and parameters $\alpha, \beta, q_{1}$ and $q_{2}$ are adjusted depending on the amplifie to match its characteristics. The AM/PM conversion of SSPA is frequently neglected, although in this paper we will take it into account for a more accurate representation. Indeed, the SSPA used in this paper is the NEC GaAs Power Amplifie utilized for standardization [52], [53] with parameters $g=19, A_{\text {sat }}=1.4, s=0.81, \alpha=-48000, \beta=0.123$, $q_{1}=3.8$ and $q_{2}=3.7$, referred to through the paper as SSPA.

In order to describe the different possible input power levels, we use the Input Back-Off (IBO) of the HPA define as

$$
I B O=10 \log _{10} \frac{P_{\text {sat }}}{P_{\text {avg }}}
$$

where the $P_{\text {sat }}$ represents the saturation input power and $P_{a v g}$ denotes the average input power of the signal at the HPA input.

Memoryless HPA Nonlinear Effects: In Figs. $1-5$, the $\mathrm{AM} / \mathrm{AM}$, the AM/PM conversion and the effect of the amplifie in the frequency-domain, respectively, are shown. It can be seen in Figs. 1 and 2 that the TWTA2 exhibits a more non-linear behavior than TWTA1 in both the AM/AM and the AM/PM characteristics. Besides, the SSPA is much more non-linear in the $\mathrm{AM} / \mathrm{AM}$ conversion whereas its behavior in $\mathrm{AM} / \mathrm{PM}$ is negligible compared to the TWTAs. Both figure have been obtained for an IBO of $2 \mathrm{~dB}$, and it can be observed that the closer to the saturation point $(0 \mathrm{~dB})$, the larger the distortions are. These effects will cause a constellation wrapping and rotation, as it will be seen in the results section.

Next, in Fig. 5, the effects of the amplifie in the frequencydomain are shown. This figur has been obtained for $N=2048$ sub-carriers, and a 64-QAM modulation. It can be seen that the signal experiences a spectral regrowth due to the nonlinearity. Again, a more accentuated nonlinear character of the amplifie

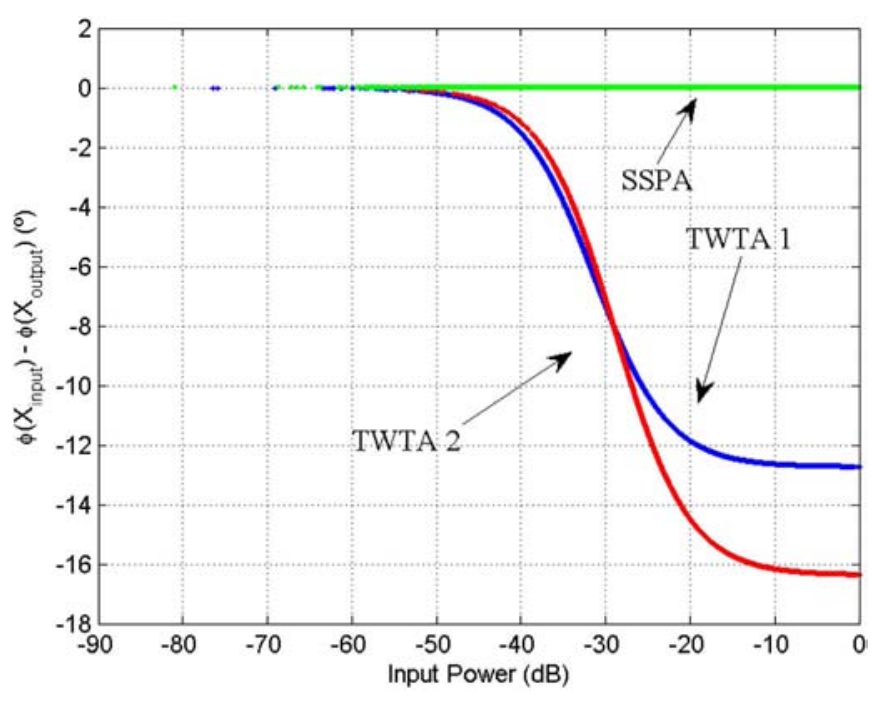

Fig. 2. HPA non-linear response for the phase. IBO $=2$ dB. TWTA1 $=$ $\alpha_{\varphi}=4, \beta_{\varphi}=9$, TWTA2 $=\alpha_{\varphi}=2$, and $\beta_{\varphi}=7$. Memoryless HPA.

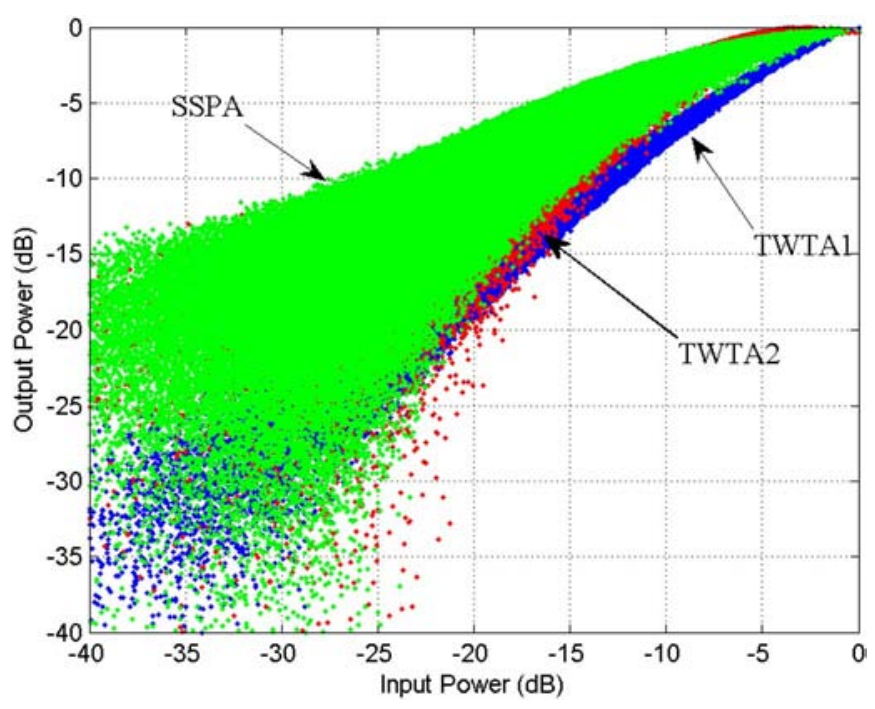

Fig. 3. HPA non-linear response for the modulo. IBO $=2$ dB. TWTA1 $=$ $\alpha_{\|}=2, \beta_{\|}=1$, TWTA2 $=\alpha_{\|}=3$, and $\beta_{\|}=2$. HPA with memory.

causes more out-band regrowth. This is especially important in broadcasting systems where the spectral masks are very strict.

HPA With Memory Nonlinear Effects: When increasing the bandwidth and the maximum transmitted power of the amplifie , memory effects can not be neglected anymore. These effects are related to frequency dependence and thermal phenomena [54]. This model can be considered as a Hammerstein system which can be modeled by a memoryless HPA followed by a linear low-pass filte [54]. In this paper, the 3-pole $(0.7692,0.1538$, 0.0769 ) low-pass filte will be considered to account for the memory of the HPA [26]. In Figs. 3 and 4, the AM/AM and AM/PM conversion for the HPA with memory are shown, respectively. It can be observed that now, instead of a bi-univocal correspondence between input and output, due to the memory, an input signal is mapped onto different points, and the function becomes a cloud.

Pre-Distortion Concept: The main idea behind the concept of pre-distortion is the aim of introducing inverse nonlinearities 


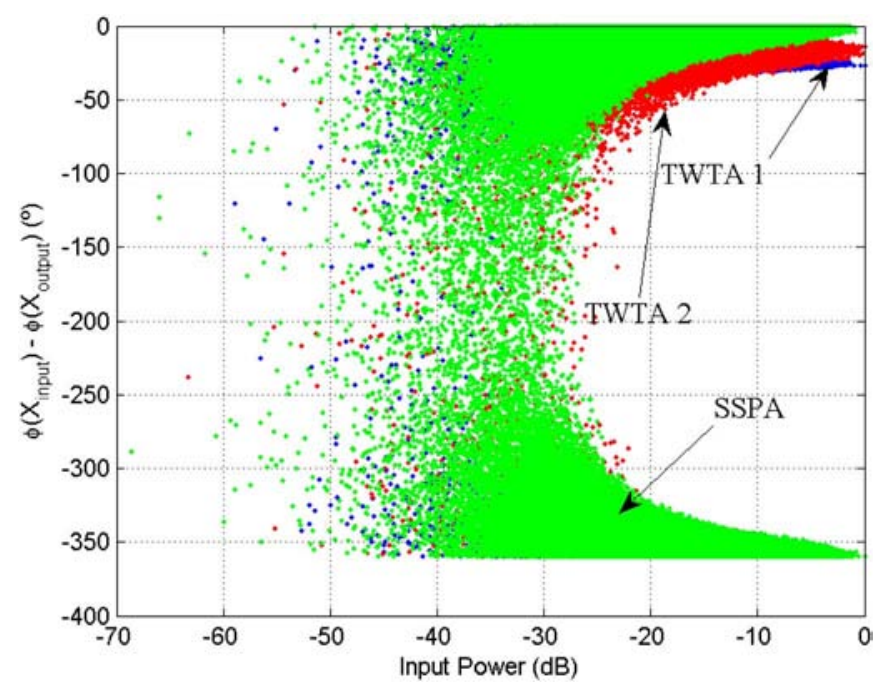

Fig. 4. HPA non-linear response for the phase. $\mathrm{IBO}=2 \mathrm{~dB}$. TWTA $1=$ $\alpha_{\varphi}=4, \beta_{\varphi}=9$, TWTA2 $=\alpha_{\varphi}=2$, and $\beta_{\varphi}=7$. HPA with memory.

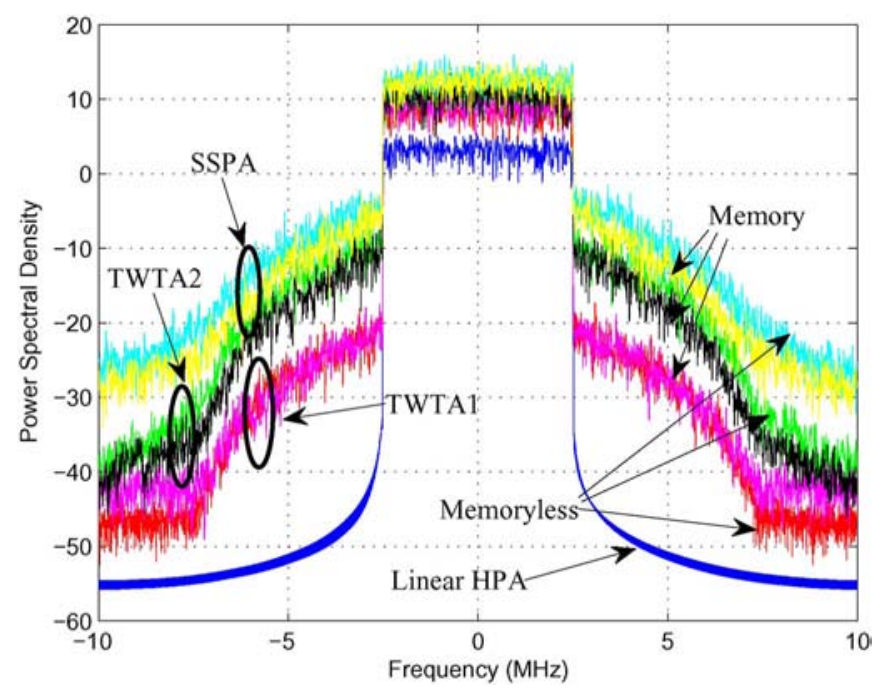

Fig. 5. HPA power spectral density. IBO $=2 \mathrm{~dB}$. TWTA $1=\alpha_{\varphi}=4$, $\beta_{\varphi}=9$, TWTA $2=\alpha_{\varphi}=2$, and $\beta_{\varphi}=7$. Memoryless and with memory.

that can compensate the $\mathrm{AM} / \mathrm{AM}$ and $\mathrm{AM} / \mathrm{PM}$ distortion of the HPA (Figs. 1 -4).

If we consider that the predistortion can be formulated in terms of an AM/AM predistortion function and an AM/PM predistortion function, the amplifie input can be re-written as

$$
y(t)=P F(|x(t)|) e^{[j P \varphi(|x(t)|)-j \phi(x(t))]}
$$

where $P F(|x(t)|)$ and $P \varphi(x(t))$ are respectively the AM/AM and the AM/PM functions of the pre-distorter. By introducing the signal $y(t)$, the pre-distorter output will be

$$
z(t)=P F(|y(t)|) e^{[j P \varphi(|y(t)|)+j \phi(y(t))]} .
$$

We would like to highlight that the examples of amplifier used in this paper are only meant to obtain results and validate the performance of the proposed pre-distorter scheme. The scheme is general and valid for any kind of amplifier Once trained with the specifi amplifie response, the ANFIS is able to pre-distort the input signal adequately and with low complexity.

\section{NEURO-FuZZY SYSTEM}

Nonlinear system identificatio has been extensively studied during the last decades and several methods and applications have been developed [55]. Multilayer feed-forward neural network is the most usual method for the identificatio of nonlinear dynamical systems [56]. Fuzzy and neuro-fuzzy models for nonlinear dynamic system identificatio have been also studied, both for forward and recurrent topologies. Several fuzzy and neuro-fuzzy models have been developed in many applications, such as process modeling, identificatio [57] and control or fault diagnosis [58]. Neural Network and fuzzy systems both are stand-alone systems. With the increase in the complexity of the process being modeled, the difficult in developing dependable fuzzy rules and membership functions increases. For these reasons, another approach has been developed, which is mostly known as neuro-fuzzy approach. It has the benefit of both, neural networks and fuzzy logic. The neuro-fuzzy system combines the advantages of fuzzy logic system, which deal with explicit knowledge that can be explained and understood, and neural networks, which deal with implicit knowledge. One of the advantages of fuzzy systems is that they describe fuzzy rules, which fi the description of real-world processes to greater extent. Another advantage of fuzzy systems is their interpretability. In turn, some of the main disadvantages of fuzzy systems are that expert's knowledge or instructions are needed in order to defin fuzzy rules, and the process of tuning of the parameters (parameters of the membership function) becomes hard. These disadvantages are related to the fact that it is not possible to train fuzzy systems. The opposite situation can be observed in the fiel of neural networks. It is possible to train the neural networks, but it is extremely difficul to use prior knowledge about the considered system and it is almost impossible to explain the behavior of the neural network system in a particular situation. In order to leverage on advantages of both systems and compensate the disadvantages, several researchers tried to combine fuzzy system with neural networks. A hybrid system called ANFIS has been proposed by [59]. Fuzzy inference in this system is realized using a training algorithm. ANFIS was firstl proposed for data predistortion in [60], although this work was focused on single-carrier QAM modulation. Next, in [43], [44], this technique is applied to single-carrier CDMA signals. Our proposal differs with the former ones in several important aspects. First, our proposal is much simpler than previous because it does not need to continue training during the running phase. The training process has been designed in such as way that the whole system is able to tackle any changes, including the memory effects, as it will be explained later in the paper, whereas in [43], [44], [60], the system is always learning for tracking changes. Second, our proposal is applied for more complex multi-carrier signals instead of single-carrier. Third, our proposal is also valid for higher order modulation. In fact, as it will be numerically shown later in the paper, the larger order modulation, the lower EVM and thus, the better performance. Fourth, our proposal is valid for memory and memoryless HPA, while the former ones are only effective for the memoryless case. 


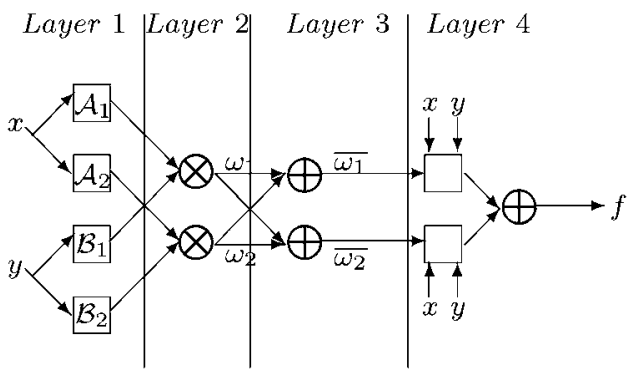

Fig. 6. Corresponding ANFIS architecture. Inputs are $x$ and $y$, whereas output is $f$.

\section{A. ANFIS Adaptive Neuro-Fuzzy Inference}

A detailed description of ANFIS can be found in [61]. The Sugeno fuzzy model was proposed by Sugeno et al. in [62]. A typical fuzzy rule in a Sugeno fuzzy model has the format

$$
\text { If } x \text { is } \mathcal{A} \text { and } y \text { is } \mathcal{B} \text { then } z=f(x, y)
$$

where $\mathcal{A}$ and $\mathcal{B}$ are fuzzy sets in the antecedent and $z=f(x, y)$ is a crisp function in the consequent. We consider the first-orde Sugeno fuzzy inference system which contains two rules

$$
\begin{aligned}
& \text { If } x \text { is } \mathcal{A}_{1} \text { and } y \text { is } \mathcal{B}_{1} \text { then } f_{1}=p_{1} x+q_{1} y+r_{1} \\
& \text { If } x \text { is } \mathcal{A}_{2} \text { and } y \text { is } \mathcal{B}_{2} \text { then } f_{2}=p_{2} x+q_{2} y+r_{2}
\end{aligned}
$$

The firin strengths $\omega_{1}$ and $\omega_{2}$ are usually obtained as the product of the membership grades in the premise part, and the output $f$ is the weighted average of each rule's output

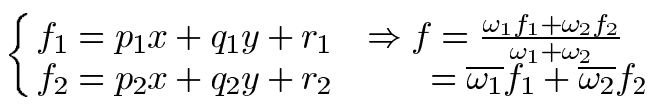

In order to facilitate the learning of the Sugeno fuzzy model, it is convenient to put the fuzzy model into framework of adaptive networks that can compute gradient vectors systematically. The resultant network architecture, called ANFIS is shown in Fig. 6, where nodes within the same layer perform functions of the same type, as detailed below $\left(\Omega_{i}^{j}\right.$ denotes the output of the $i$ th node in $j$ th layer) [61].

Layer 1: Each node in this layer generates membership grades of linguistic label. For instance, the node function of the $i$ th node may be a generalized Gaussian membership function

$$
\Omega_{i}^{1}=\mu_{A_{i}}(x)=\exp \left(-\frac{\left(x-c_{i}\right)^{2}}{2 \sigma_{i}^{2}}\right)
$$

where $x$ is the input to node $i, \mathcal{A}_{i}$ is the linguistic label (small, large, etc.) associated with this node, and $\left\{c_{i}, \sigma_{i}, \beta_{i}\right\}$ is the parameter set that changes the shapes of the membership function. Parameters in this layer are referred to as the premise parameters.

Layer 2: Each node in this layer calculates the firin strength of a rule via multiplication

$$
\Omega_{i}^{2}=\mu_{\mathcal{A}_{i}}(x) \times \mu_{\mathcal{B}_{i}}(x), \quad i=1,2
$$

Layer 3: Node $i$ in this layer calculates the ratio of the $i$ th rule's firin strength to the sum of all firin strengths

$$
\Omega_{i}^{3}=\overline{\omega_{i}}=\frac{\omega_{i}}{\omega_{1}+\omega_{2}}, \quad i=1,2
$$

Layer 4: Node $i$ in this layer computes the contribution of $i$ th rule toward the overall output, with the following node function

$$
\Omega_{i}^{4}=\overline{\omega_{i}} f_{i}=\overline{\omega_{i}}\left(p_{i} x+q_{i} y+r_{i}\right)
$$

Where $\overline{\omega_{i}}$ is the output of layer 3 , and $p_{i}, q_{i}, r_{i}$ is the parameter set. Parameters in this layer will be referred to as the consequent parameters.

Layer 5: The single node in this layer computes the overall output as the summation of contributions from each rule

$$
\Omega_{i}^{5}=f=\Sigma_{i} \overline{\omega_{i}} f_{i}
$$

\section{B. ANFIS Learning Algorithm}

From the ANFIS architecture in Fig. 6, it is observed that given the values of premise parameters $\left\{c_{i}, \sigma_{i}, \beta_{i}\right\}$, the overall output $f$ can be expressed as linear combinations of the consequent parameters $p_{i}, q_{i}, r_{i}$

$$
\begin{aligned}
f= & \overline{\omega_{1}} f_{1}+\overline{\omega_{2}} f_{2} \\
= & \overline{\omega_{1}}\left(p_{1} x+q_{1} y+r_{1}\right)+\overline{\omega_{2}}\left(p_{2} x+q_{2} y+r_{2}\right) \\
= & \left(\overline{\omega_{1}} x\right) p_{1}+\left(\overline{\omega_{1}} y\right) q_{1}+\overline{\omega_{1}} r_{1} \\
& +\left(\overline{\omega_{2}} x\right) p_{2}+\left(\overline{\omega_{2}} y\right) q_{2}+\overline{\omega_{2}} r_{2}
\end{aligned}
$$

The ANFIS learning algorithm consists of adjusting the above set of parameters. To that end a mixture of backpropagation and Least Squares Estimation (LSE) is used. Backpropagation is used to learn the premise parameters $\left\{c_{i}, \sigma_{i}, \beta_{i}\right\}$ and LSE is used to determine the parameters in the rule's consequents $p_{i}$, $q_{i}, r_{i}$. Every step in the learning procedure has two passes. In the forward pass, the network inputs propagate forward until layer 4, and the consequent parameters are estimated by LSE method, while the premise parameters remain fi ed. In the backward pass, the error signals are backward propagated, and the premise parameters are updated by gradient descent, while consequent parameters remain fi ed. This hybrid algorithm is used for training the Fuzzy Inference Systems (FIS) membership function parameters, in order to model a given set of input/output data.

\section{Evaluation of Training Parameters}

One of the goals of the predistorter design was the low complexity in the running phase, and thus, the adaptive training during this phase should be avoided. For this reason, our ANFIS has been designed in such as way it is able to generalize for tracking changes in the HPA and also the memory effects. In order to do that, cross validation and the evaluation of the main parameters (i.e., the number of epochs and number of rules) in the design is shown in the following. First, the impact of the number of training epochs on the training error, is evaluated. It can be seen in Fig. 7(a), where the training error is plotted as a function of the number of epochs, that this parameter is not especially critical since, once the number of epochs is enough for 


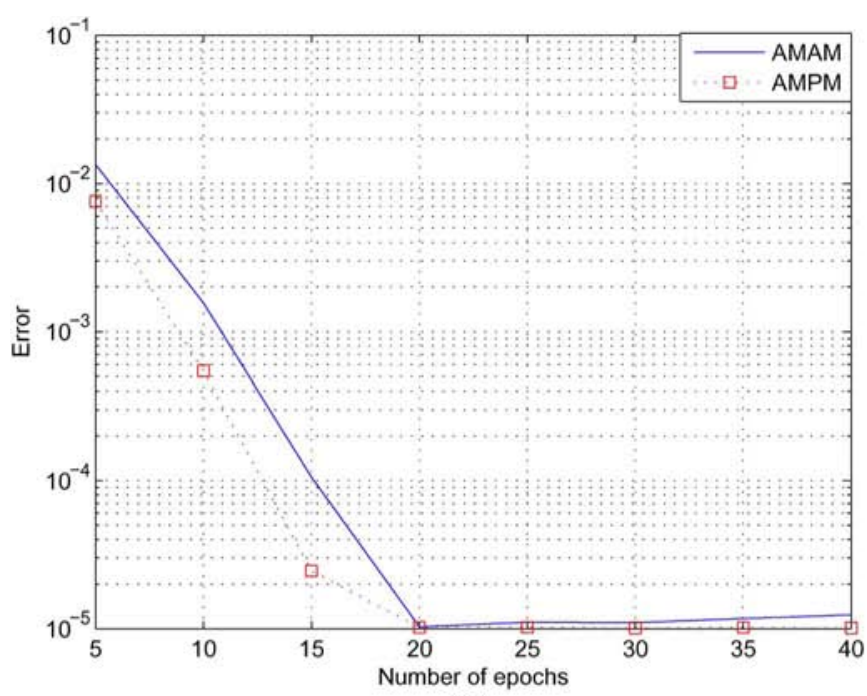

(a)

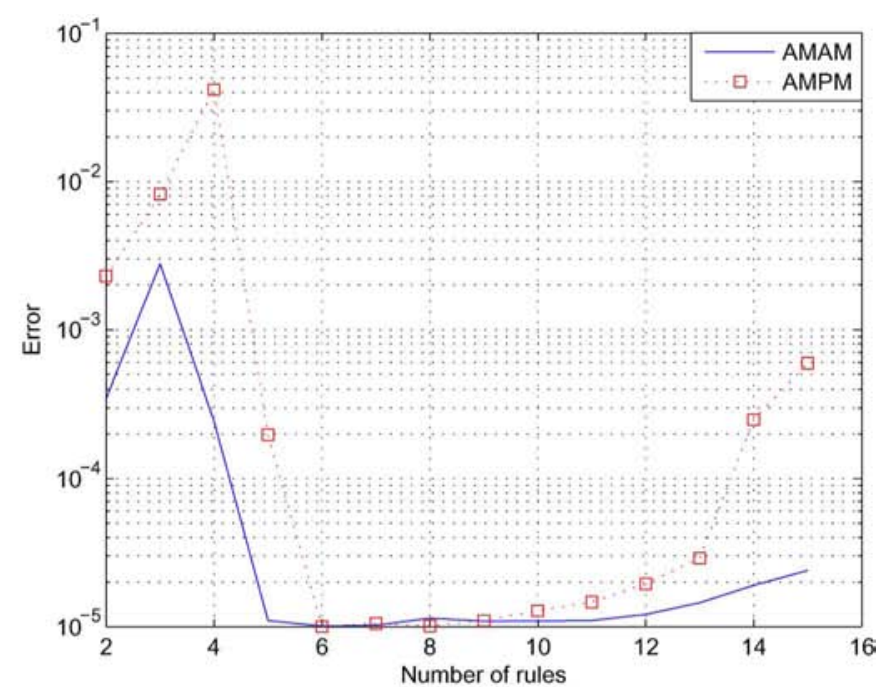

(b)

Fig. 7. Training error for different parameters. (a) Number of epochs. (b) Number of rules.

training the ANFIS, the performance remains similar when increasing its number. The training error shown here is obtained using unseen samples and for both effects, the Amplitude-toAmplitude and the Amplitude-to-Phase distortion. It can be observed however that, once the number of epochs reaches a goodenough value, as the number of epochs increases so slightly the error, indicating that the systems begins to be over-trained. More influenc exhibits the number of rules. In Fig. 7(b), the training error is shown depending on the number of rules used in the design of ANFIS. It can be seen that between fi e and ten, the best performance is obtained. After that, the system is too much over-fitte and it does not properly generalize. Besides, it can be observed that the AM/PM distortion is more sensitive to the number of rules. It should be also noted that the rules have been limited to the simplest ones, again for obtaining a powerful generalization.

For these reasons, our design uses fi e and six simple and rules for $\mathrm{AM} / \mathrm{AM}$ and $\mathrm{AM} / \mathrm{PM}$ functions, respectively, and we have trained them by using 20 epochs.

\section{Predistorter Architecture}

During the off-line training process, the neuro-fuzzy pre-distorter model samples the amplifie data and recovers the amplitude and phase of the input signals. Next, the firs neuro-fuzzy $\mathcal{A N} \mathcal{F} \mathcal{I S} 1$ used to approximate the pre-distorter $\mathrm{AM} / \mathrm{AM}$ function $P F(|x(t)|)=F^{-1}(|x(t)|)$, is generated by applying the HPA output amplitude $|y(t)|$ as the training input of the neuro-fuzzy scheme, while the input amplitude of HPA $|x(t)|$ is provided as the output training data. When the neuro-fuzzy training converges, its response will be very close to the inverse of the AM/AM HPA function. The second neuro-fuzzy $\mathcal{A N} \mathcal{F} \mathcal{I} S 2$ uses the input amplitude signal of the HPA as training input data, while the output training data are the phase differences between the input and output signals of the HPA. When this neuro-fuzzy system converges, it approximates the AM/PM HPA function $P \varphi(|x(t)|)=-\varphi[P F(x(t))]=\varphi\left[F^{-1}(|x(t)|)\right]$. Once the $\mathcal{A N} \mathcal{F} \mathcal{I} S 1$ and $\mathcal{A N} \mathcal{F} \mathcal{I} S 2$ models have been obtained, in the

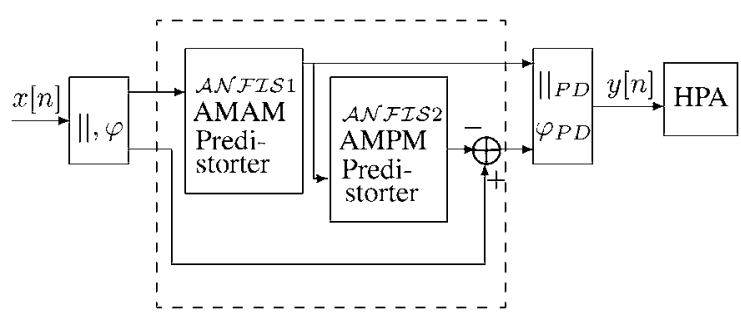

Fig. 8. Implemented ANFISs architecture.

real-time running operation, the modulo and the phase of the modulated signal $x[n]$ is computed. The modulo is introduced into the $\mathcal{A N} \mathcal{F} \mathcal{I} S 1$ and its output is fed to the $\mathcal{A N F} \mathcal{F} \mathcal{S} 2$ and to the output modulo of the signal. Next, the output of $\mathcal{A N} \mathcal{F} \mathcal{I S} 2$ is subtracted from the original phase and this signal feeds the output phase block. This modulo-phase block will generate the fina predistorted signal $y[n]$ to be amplifie by the HPA. The architecture of the neuro-fuzzy predistorter in the running phase is shown in Fig. 8.

\section{E. Complexity Analysis}

In this section, the complexity analysis will be described and compared to other already existing methods based on the number of real operations needed for the algorithms. Our proposal needs $4 \times N_{\text {rules }}, 2 \times\left(2 \times N_{\text {rules }}-1\right)$ and $2 \times N_{\text {rules }}$ real products, additions and divisions, respectively. All these numbers are operations per sample (i.e., for an OFDM symbol with $\mathrm{N}$ sub-carriers, these numbers need to be multiplied by N). Since our ANFIS uses 5 rules (i.e., $N_{\text {rules }}=5$ ), the total number of operations are 20,18 and 10, respectively. On the other hand, the number of operations of other schemes such as the polynomial-based depends on different parameters. In [23], where the polynomial is reduced to a couple of dimensions ( $N_{A}$ and $N_{\Theta}$ are, respectively, the number of terms in the polynomial for the amplitude and the phase, and $N_{e}$ is the number of terms for the exponential values), the number of operations are shown in Table I. The complexity for the proposal in [24] 
TABLE I

COMPlEXITy SUMmARY ANALYSIS PER SAMPLE. N $=2048$

\begin{tabular}{|c|c|c|c|c|c|c|c|c|c|c|}
\hline Description & Proposal & $\begin{array}{l}\text { PD in [23]. } \\
N_{A}=N_{\Theta}= \\
N_{e}=2\end{array}$ & TOD & FOD & $\begin{array}{l}\text { PD in [24]. } \\
T=8192\end{array}$ & $\begin{array}{l}\text { SCPWL } \\
\text { in [25] }\end{array}$ & $\begin{array}{l}\text { Secant } \\
\text { in }[25]\end{array}$ & $\begin{array}{l}\text { PD in [40]. } \\
23 \text { neurons. } \\
Q=N\end{array}$ & $\begin{array}{l}\text { PD in } \\
{[41] . M=} \\
8, L=N\end{array}$ & $P D$ in $[36]$ \\
\hline \# Mults & 20 & $\begin{array}{l}9+N_{A}+ \\
N_{\Theta}=13\end{array}$ & 8 & 10 & $\begin{array}{l}\mathcal{O}(T)+ \\
\mathcal{O}\left(N \log _{2} N\right)\end{array}$ & 11 & 15 & $\begin{array}{l}Q \times \\
\left(N_{\text {neurons }}+\right. \\
1)=49152\end{array}$ & $\begin{array}{l}M \times L= \\
16384\end{array}$ & 10 \\
\hline \# Adds & 18 & 8 & 8 & 10 & $\mathcal{O}(T)$ & 11 & 18 & $\begin{array}{l}N_{\text {neurons }}+ \\
1=24\end{array}$ & $\begin{array}{l}M \times L= \\
16384\end{array}$ & 10 \\
\hline \# Divs. & 10 & 0 & 0 & 0 & 0 & 0 & 3 & $\begin{array}{l}N_{\text {neurons }} \\
1=24\end{array}$ & $\begin{array}{l}M \times L= \\
16384\end{array}$ & 0 \\
\hline Type & ANFIS & Poly & Poly & Poly & Poly & Poly & Poly & NN & NN & LUT - Poly \\
\hline $\begin{array}{l}\text { HPA (M } \\
\text { or ML) }\end{array}$ & $\mathrm{M}$ & ML & ML & ML & $\mathrm{M}$ & ML & ML & $\mathrm{M}$ & ML & ML \\
\hline
\end{tabular}

is $\mathcal{O}(T)+\mathcal{O}\left(N \log _{2} N\right)$, where $T$ is the number of samples for training and its high complexity is due to the FFT. Another two polynomial-based schemes are proposed in [25], namely, Simplicial Canonical Piecewise Linear (SCPWL) and Secant (based on secant curve). Those methods exhibit low number of operations in the running phase, although their performance is compromised. As a reference, the complexity of the Third Order Distortion (TOD) and Fifth Order Distortion (FOD) polynomial-based are also included.

Two NN-based algorithms have been analyzed. The one proposed in [40], whose complexity depends on the number of hidden neurons and the number of inputs (usually the number of sub-carriers), and the one in [41], with larger complexity but better performance. Finally, also a joint LUT-based and polynomial-based method is analyzed, with reduced complexity and reasonable results.

In Table I, the complexity comparison is summarized for different Pre-distorters (PD) in the literature. The number of operations is calculated for the specifi configuratio and per sample. The number of sub-carriers is $N=2048$. It can be observed that the complexity of our proposal is comparable (although slightly higher) to the polynomial-based schemes but with much better performance, as it will be seen in next sections. Besides, our proposal achieves better performance than NN-based methods with much lower complexity, and even in scenarios with memory.

\section{ERRor Vector MAgnitude (EVM)}

The EVM is a common figur of merit for assessing the quality of digitally modulated signals. It accounts for the difference between the expected complex voltage value of a demodulated symbol and the value of the current received symbol. It is very useful for microwave engineers because it contains information about amplitude and phase errors in the signal [63]. The average EVM is define as

$$
E V M=\frac{1}{N_{s}} \frac{1}{N} \sqrt{\frac{\frac{1}{M} \sum_{r=1}^{M}\left|S_{\text {ideal }}^{r}-S_{\text {measured }}^{r}\right|^{2}}{\frac{1}{M} \sum_{r=1}^{M}\left|S_{\text {ideal }}^{r}\right|^{2}}}
$$

where $S_{\text {ideal }}^{r} \in \mathbb{C}$ is the $r$ th point out of $M$ for the ideal constellation in a $M$-QAM modulation, and $S_{\text {measured }}^{r}$ is the measured $r$ th constellation point after the HPA. $\mathbf{S}_{\text {ideal }}$ is a vector containing all the ideally transmitted complex symbols.

The distributions of the real and imaginary parts of $\mathbf{S}_{\text {ideal }}$ are both a discrete uniform (if $\log _{2}(M)$ is a power of two), with values from $-\sqrt{M}+1$ to $\sqrt{M}-1$ in steps of 2 . Since our models are based on Gaussian membership functions (see eq. (12)), the obtained $\mathbf{S}_{\text {measured }}$ constellation (i.e., $\mathbf{S}_{A N F I S}$ ) will exhibit a distribution similar to $\mathbf{S}_{\text {ideal }}$ with a Gaussian distribution around each discrete value. Thus, the difference between both will be Gaussian, i.e.,

$$
\mathbf{S}_{\text {err, real }}=\mathcal{R}\left\{\mathbf{S}_{\text {ideal }}-\mathbf{S}_{\text {ANFIS }}\right\} \approx \mathcal{N}\left(0, \sigma_{i}^{2}\right)
$$

and

$$
\mathbf{S}_{\text {err }, \text { imag }}=\mathcal{I}\left\{\mathbf{S}_{\text {ideal }}-\mathbf{S}_{\text {ANFIS }}\right\} \approx \mathcal{N}\left(0, \sigma_{i}^{2}\right)
$$

Therefore, the absolute value $\left|\mathbf{S}_{\text {err }}\right|=\sqrt{\mathbf{S}_{\text {err,real }}^{2}+\mathbf{S}_{\text {err }, \text { imag }}^{2}}$ will exhibit a Rayleigh distribution of parameter $\sigma_{r}=\sigma_{i}$. Thus, the distribution of $\left|\mathbf{S}_{e r r}\right|^{2}$ will be the Gamma distribution $(\Gamma)$ with shape and scale parameters equal to 1 and $2 \sigma_{i}^{2}$, respectively. Applying the Central Limit Theorem to (18) we obtain that the average EVM can be estimated as follows

$$
\overline{E V M}=\frac{1}{N_{s}} \frac{1}{N} \sqrt{\frac{3 M \sigma_{i}^{2}}{(M-1)}}
$$

since $1 / M \sum_{r=1}^{M}\left|S_{\text {ideal }}^{r}\right|^{2}$ is the mean energy of the constellation and can be calculated as $2 / 3(M-1)$ and $\sum_{r=1}^{M} \mid S_{\text {ideal }}^{r}$ $\left.S_{A N F I S}^{r}\right|^{2} \rightarrow M \times \mathrm{E}\left\{\left|S_{\text {ideal }}^{r}-S_{A N F I S}^{r}\right|^{2}\right\}$ according to the Central Limit Theorem, and the expectation of a Gamma distribution with shape equal to 1 and scale $2 \sigma_{i}^{2}$ is $2 \sigma_{i}^{2}$. In Table II, the obtained EVM by simulations and by using our analytical calculation are summarized for the different HPAs evaluated in the paper. Two conclusions can be extracted from these data. First, the analytical expression is accurate enough and second, the EVM of our proposed scheme is very low.

\section{RESULTS}

The three different amplifier presented in the paper have been simulated (two TWTA and one SSPA), all of them both with and without memory. The number of sub-carriers has been fi ed to $N=2048$ (typical value for DVB-T or DAB standards). The modulation was 16-QAM and 64-QAM, i.e., $M=16$ and $M=64$, respectively. The results have been obtained with a fourfold oversampling factor. In Figs. 9 and 10, the transmitted constellation after the TWTA1 and the SSPA are shown, respectively for 64-QAM modulation. It can be seen that, without the predistortion, the constellation is rotated and wrapped. It can also be observed in Fig. 10 that the SSPA distorts more the 
TABLE II

ANALYTICAL AND SimULATION RESULTS FOR THE EVM. 64-QAM

\begin{tabular}{|c|c|c|c|c|}
\hline Amplifier & Memory & $\sigma_{i}$ & EVM (An.) & EVM (Sim.) \\
\hline TWTA1 & No & $8.6 \cdot 10^{-3}$ & $8.76 \cdot 10^{-7}$ & $9.07 \cdot 10^{-7}$ \\
\hline TWTA1 & Yes & $8.7 \cdot 10^{-3}$ & $8.87 \cdot 10^{-7}$ & $9 \cdot 10^{-7}$ \\
\hline TWTA2 & No & $8.5 \cdot 10^{-3}$ & $8.72 \cdot 10^{-7}$ & $9 \cdot 10^{-7}$ \\
\hline TWTA2 & Yes & $4.05 \cdot 10^{-2}$ & $4.14 \cdot 10^{-6}$ & $4.31 \cdot 10^{-6}$ \\
\hline SSPA1 & No & $6.62 \cdot 10^{-2}$ & $6.77 \cdot 10^{-6}$ & $7.01 \cdot 10^{-6}$ \\
\hline SSPA 1 & Yes & $6.18 \cdot 10^{-2}$ & $6.32 \cdot 10^{-6}$ & $6.56 \cdot 10^{-6}$ \\
\hline
\end{tabular}

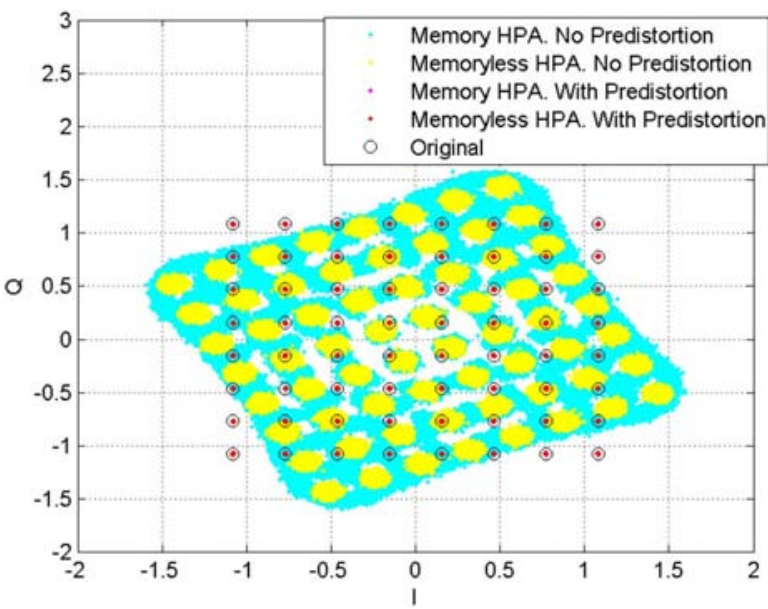

Fig. 9. Constellation after memory and memoryless TWTA 1 HPA, with and without predistortion. 64-QAM.

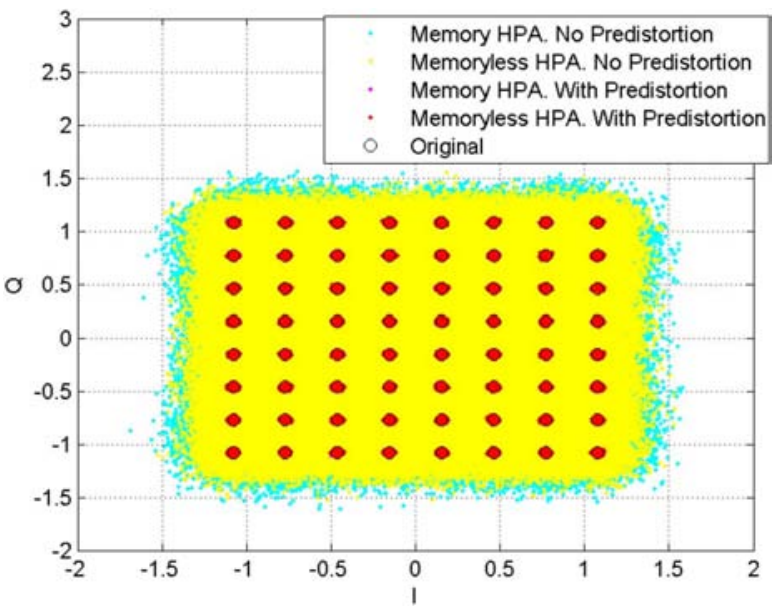

Fig. 10. Constellation after memory and memoryless SSPA 1 HPA, with and without predistortion. 64-QAM.

constellation than the TWTAs. In all cases, the memory of the HPA also introduces an interdependence between constellation points. However, by using our proposal, the constellation points are practically restored to their original locations. Thus, as it can be seen in Fig. 11, where the BER has been plotted for 64-QAM modulation, the BER degradation is negligible. In this figur the performance results using our proposal for TWTA1, TWTA2 and SSPA have been plotted. For comparison purposes, also the performance obtained by two LUT-based methods such as [30] and [35], a NN-based technique in [38] and a polynomial solution in [23] have been drawn. The BER obtained with our ANFIS proposal after TWTA1, TWTA2 and SSPA with and

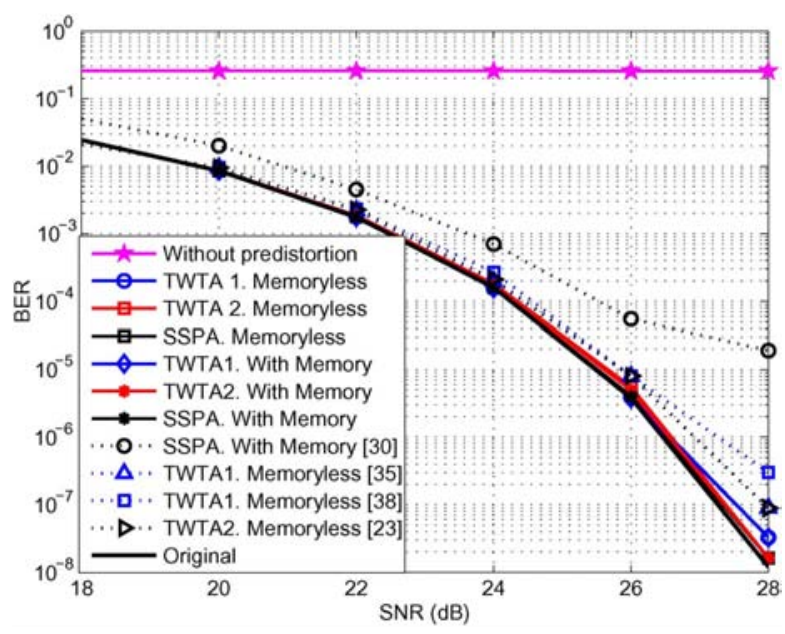

Fig. 11. Bit Error Rate. 64-QAM. TWTA1, TWTA2, and SSPA. Memoryless and memory HPA.

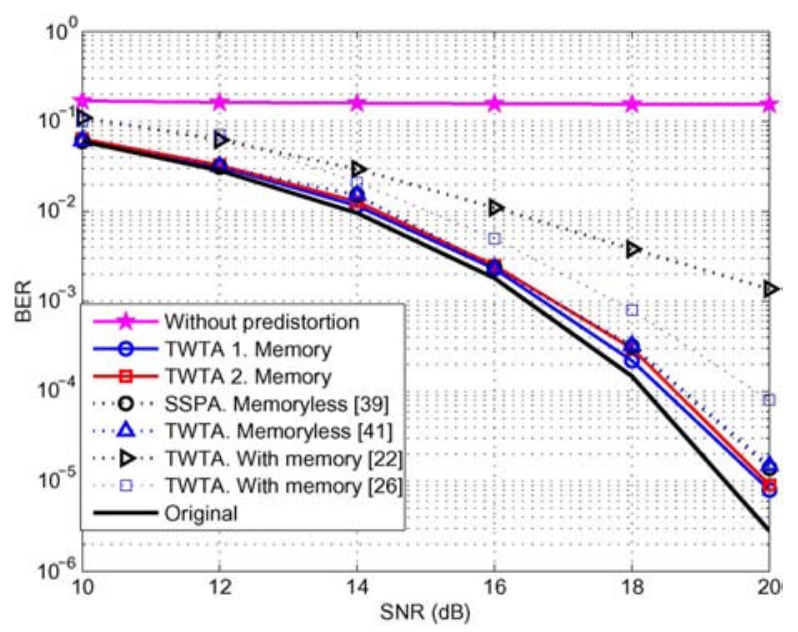

Fig. 12. Bit Error Rate. 16-QAM. TWTA1, TWTA2, and SSPA. Memoryless and memory HPA.

without memory is very close to the BER obtained with the original signal without distortion (less than $0.1 \mathrm{~dB}$ of performance degradation). On the other hand, references [30], [35], [38] and [23] are not able to completely compensate the distortion and there is a non-negligible performance degradation, more noticeable for the case of SSPA with memory. Indeed, for the scenario with memory, degradation by using the LUT-based in [30] is more than $2 \mathrm{~dB}$ whereas for the memoryless scenarios, approaches in [35] (LUT-based), [38] (NN-based) and [23] (polynomial-based) the degradation is around $1 \mathrm{~dB}$ for large SNR. Next, in Fig. 12, the performance for 16-QAM modulation of our proposal, two other NN-based techniques in [39], [41] and two other polynomial-based schemes in [22], [26] is plotted. Several conclusions can be extracted from this figure The firs one is that our proposal outperforms the others. Indeed, even when applied to scenarios with memory, our proposal obtains better performance than NN-based approaches applied to memoryless amplifiers basically because our proposal exploits the powerful fuzzy rules. Second, the polynomial proposals for scenarios with memory are not able to completely linearize the 


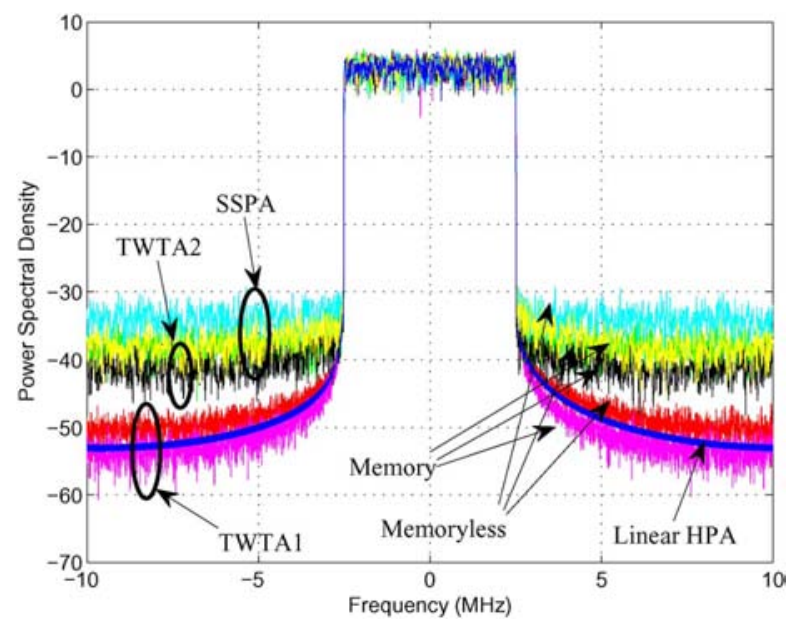

Fig. 13. Power spectral density after memory and memoryless HPA with the ANFIS predistorters.

HPA and exhibit a larger (compared to our method) performance degradation. In fact, these methods, compared to our proposal are more than $1 \mathrm{~dB}$ worse and this gap increases with the signal to noise ratio because these schemes suffer from an error floo for large SNR. Third, it can be seen that our proposal exhibits better performance for 64-QAM than for 16-QAM, as expected according to (21). In Fig. 13, the power spectral density of the signals after the HPA and previously predistorted with our proposal are shown. Since TWTA1 is less nonlinear than TWTA2 and SSPA, the spectral regrowth (measured with respect to the original spectrum) after our scheme is almost negligible, whereas for the TWTA2 or SSPA it is about $8 \mathrm{~dB}$, small value compared to $40 \mathrm{~dB}$ without pre-distortion and valid for fulfillin the strict spectral mask in broadcasting systems such as [1] or [2].

\section{CONCLUSION}

In this paper an efficien and effective HPA predistorter based on ANFIS with application to broadcasting of high order modulated OFDM signals has been presented and analyzed. It has been shown that the proposal, once it has been trained and without any further requirement, is able to predistort the signal in such as way that the output of the HPA is extremely similar to the original signal, and thus, the BER performance loss is negligible. Besides, results allow us to conclude that our proposal is valid for memory and memoryless HPA, obtaining similar results in both scenarios. Moreover, it offers a better BER performance than what can be achieved with other methods in the literature. Once trained, the complexity of the proposal is reduced and thus, it can be implemented at low cost with reduced-size electronics. For these reasons, we believe it is a very good candidate to be used for broadcasting systems.

\section{REFERENCES}

[1] "Digital video broadcasting (DVB): Implementation guidelines for DVB terrestrial services: Transmission aspects," ETSI TR 101190 V.1.2.1, 2004, Technical report.

[2] "Digital audio broadcasting (DAB): Guidelines and rules for implementation and operation: Part I, II and III," ETSI TR 101 496-1,2,3 V 1.1.1, 2001, Technical report.

[3] "Digital radio mondiale (DRM): System specification" ETSI TS 101 980 V1.1.1 (2001-09), 2001.
[4] IEEE Standard for Local and Metropolitan Area Networks Part 16: Air Interface for Fixed Broadband Wireless Access Systems, IEEE 802.162004, 2004, Technical report, IEEE.

[5] IEEE Standard for Local and Metropolitan Area Networks Part 16: Air Interface for Fixed and Mobile Broadband Wireless Access Systems. Amendment 2: Physical and Medium Access Control Layers for Combined Fixed and Mobile Operation in Licensed Bands and Corrigendum 1, IEEE 802.16e-2005, 2005, Technical report.

[6] L. Cimini, Jr. and N. R. Sollenberger, "Peak-to-average power ratio reduction of an OFDM signal using partial transmit sequences," IEEE Commun. Lett., vol. 4, no. 3, pp. 86-88, Mar. 2000.

[7] A. D. S. Jayalath and C. Tellambura, "SLM and PTS peak-power reduction of OFDM signals without side information," IEEE Trans. Wireless Commun., vol. 4, no. 5, pp. 2006-2013, Sep. 2005.

[8] R. W. Bauml, R. F. H. Fisher, and J. B. Huber, "Reducing the peak-toaverage power ratio of multicarrier modulation by selected mapping," IEE Electron. Lett., vol. 32, no. 22, pp. 2056-2057, Oct. 1996.

[9] M. Breiling, S. H. Muller-Weinfurtner, and J. B. Huber, "SLM peakpower reduction without explicit side information," IEEE Commun. Lett., vol. 5, no. 6, pp. 239-241, Jun. 2001.

[10] J. Tellado, "Peak-to-average power reduction," PhD thesis, Standford University, , Sep. 1999.

[11] J. A. Davis and J. Jedwab, "Peak-to-mean power control in OFDM, Golay complementary sequences and Reed-Muller codes," IEEE Trans. Inf. Theory, vol. 45, no. 7, pp. 2397-2417, Nov. 1999.

[12] V. P. G. Jiménez, M. J. Fernández-Getino García, M. P. Sánchez Fernández, and A. G. Armada, "Efficien implementation of complementary Golay sequences for PAR reduction and forward error correction in OFDM-based WLAN systems," AEÜ-Int. J. Electron. Commun., vol. 62 , no. 9 , pp. $683-694$, Oct. 2008

[13] R. F. H. Fischer and C. Siegl, "Reed-Solomon and simplex codes for peak-to-average power ratio reduction in OFDM," IEEE Trans. Inf. Theory, vol. 55, no. 4, pp. 1519-1528, Apr. 2009.

[14] P. Van Eetvelt, G. Wade, and M. Tomlinson, "Peak to average power reduction for OFDM schemes by selective scrambling," IEE Electron. Lett., vol. 32, no. 21, pp. 1963-1964, Oct. 1996.

[15] A. D. S. Jayalath and C. Tellambura, "Reducing the peak-to-average power ratio of orthogonal frequency division multiplexing signal through bit or symbol interleaving," IEE Electron. Lett., vol. 36, no. 13, pp. 1161-1163, Jun. 2000.

[16] D. Wilich, N. Dinur, and A. Glinowiecki, "Level clipped high-order OFDM," IEEE Trans. Commun., vol. 48, no. 6, pp. 928-930, Jun. 2000.

[17] X. Li and L. J. Cimini, "Effect of clipping and filterin on the performance of OFDM," IEEE Commun. Lett., vol. 2, no. 5, pp. 131-133, May 1998.

[18] B. S. Krongold and D. L. Jones, "PAR reduction in OFDM via active constellation extension," IEEE Trans. Broadcast., vol. 49, no. 3, pp. 258-268, Sep. 2003.

[19] Y. Jabrane, V. P. G. Jiménez, A. G. Armada, B. Ait Es Said, and A. Ait Ouahman, "Reduction of the envelope fluctuation of multi-carrier modulations using neural fuzzy systems," IEEE Trans. Commun., 2009, In revision.

[20] S. A. Aburakhia, E. F. Badran, and D. A. E. Mohamed, "Linear companding transform for the reduction of peak-to-average power ratio of OFDM signals," IEEE Trans. Broadcast., vol. 55, no. 1, pp. 155-160, Mar. 2009.

[21] A. Bo, Y. Zhi-xing, P. Chang-yong, Z. Tao-tao, and G. Jian-hua, "Effects of PAPR reduction on HPA predistortion," IEEE Trans. Consum. Electron., vol. 51, no. 1, pp. 1143-1147, Nov. 2005.

[22] J. Li and J. Ilow, "Adaptive Volterra predistorters for compensation of non-linear effects with memory in OFDM transmitters," in Proc. IEEE 4th Annu. Commun. Netw. Services Res. Conf. (CNSR), 2006, pp. 103-106.

[23] A. N. D'Andrea, V. Lottici, and R. Reggianini, "RF power amplifie linearization through amplitude and phase predistortion," IEEE Trans. Communications, vol. 44, no. 11, pp. 1477-1484, November 1996.

[24] A. B. J. Kokkeler, "A crosscorrelation predistorter using memory polynomials," in Proc. Int. Symp. Circuits Syst. (ISCAS), 2004, vol. 3, pp. 345-348, IEEE

[25] M. Y. Cheong, E. Aschbacher, P. Brunmayr, and T. Laakso, "Comparison and experimental verificatio of two low-complex digital predistortion methods," in 39th Asilomar Conf. Signals, Syst., Comput., 2005.

[26] T. Wang and J. Ilow, "Compensation of nonlinear distortions with memory effects in OFDM transmitters," in Proc. IEEE Global Telecommun. Conf. (GLOBECOM), Dallas, Tex, USA, Dec. 2004, vol. 4, pp. 2398-2403. 
[27] L. Ding, G. T. Zhou, D. R. Morgan, Z. Ma, J. S. Kenney, J. Kim, and C. R. Giardina, "A robust digital baseband predistorter constructed using memory polynomials," IEEE Trans. Commun., vol. 52, no. 1, pp. 159-165, Jan. 2004.

[28] C. Yu, Y. Liu, and S. Li, "Triangular memory polynomial predistorter," in Proc. 5th Int. Conf. Wireless Commun., Netw., Mobile Comput. (WiCom), 2009, pp. 1-4.

[29] K. Wesolowski, "A novel fast HPA predistorter for high PAPR signals," in Proc. IEEE 16th Int. Symp. Pers., Indoor, Mobile Radio Commun. (PIMRC), Berlin, Sep. 2005, pp. 863-867.

[30] H. (Terry Hoh) Zhi-yong, G. Jian-hua, G. Shu-jian, and W. Gang, "An improved look-up table predistortion technique for HPA with memory effects in OFDM systems," IEEE Trans. Broadcast., vol. 52, no. 1, pp. 87-91, Mar. 2006.

[31] B. Ai, Z. X. Yang, C. Y. Pan, S. G. Tang, and T. T. Zhang, "Analysis on LUT based predistortion method for HPA with memory," IEEE Trans. Broadcast., vol. 53, no. 1, pp. 127-131, Mar. 2007.

[32] L. H. Tran, T. V. Mai, and J. A. Molnar, "Embedded system to characterize HPA and calculate nonlinear predistortion neural network's weights for HPA," in Proc. IEEE Int. Instrum. Meas. Technol. Conf. (IMTC), Como, Italy, May 2004, pp. 424-428.

[33] L. Gatet, F. Bony, H. Tap-Béteille, E. Moutaye, F. Jayat, D. Roviras, and F. Gizard, "Embedded updating system based on integrated NNs in order to achieve adaptative predistortion of nonlinear HPA characteristics," in Proc. IEEE Int. Instrum. Meas. Technol. Conf. (I2MTC), Singapore, May 2009, pp. 1-5.

[34] S. Hu, G. Wu, J.-J. Ping, and S.-Q. Li, "HPA nonlinearity reduction by joint predistorter and tone-reservation with null subcarriers in WiMAX systems," in In Proc. 4th IEEE Int. Conf. Circuits Syst. for Commun. (ICCSC), Shangai, May 2008, pp. 187-190.

[35] T. M. Nguyen, J. Yoh, C. H. Lee, H. T. Tran, and D. M. Johnson, "Modeling of HPA and HPA linearization through a predistorter: Global broadcasting service applications," IEEE Trans. Broadcast., vol. 49, no. 2, pp. 132-141, Jun. 2003.

[36] H.-H. Chen, C.-H. Lin, P.-C. Huang, and J.-T. Chen, "Joint polynomial and look-up-table predistortion power amplifie linearization," IEEE Trans. Circuits Syst. II: Expr. Briefs, vol. 53, no. 8, pp. 612-616, Aug. 2006.

[37] H.-G. Ryu, T.P. Hoa, K.M. Lee, S.-W. Kim, and J.-S. Park, "Improvement of power efficien y of HPA by the PAPR reduction and predistortion," IEEE Trans. Consum. Electron., vol. 50, no. 1, pp. 119-124, Feb. 2004.

[38] R. Zayani, R. Bouallegue, and D. Roviras, "Adaptive predistortions based on neural networks associated with Levenberg-Maquard algorithm for satellite downlinks," EURASIP J. Wireless Commun., Netw. vol. 2008, p. 15, 2008.

[39] W. Henghui, Z. Hao, Y. Wu, and F. Suili, "Neural network predistortion based on error-filterin algorithm in OFDM system," in In Proc. IEEE Int. Conf. Wireless Commun., Netw., Mobile Comput., 2005, vol. 1, pp. 193-196.

[40] J. Yang, J. Gao, X. Deng, and M. Yang, "Predistortion of nonlinear high-power amplifier using neural networks," in 9th Int. Conf. Signal Process. ICSP, 2008, pp. 1695-1698, 26-29.

[41] N. Rodriguez and C. Cubillos, "Orthogonal neural network based predistortion for OFDM systems," in In Proc. IEEE Electron., Robot. Autom. Mechan. Conf. CERMA, 2007, pp. 225-228.

[42] J. S. R. Jang, "Adaptive-network-based fuzzy inference systems," IEEE Trans. Syst., Man, Cybern., vol. 23, no. 3, pp. 665-685, 1993.

[43] K. C. Lee and P. Gardner, "A novel digital predistorter technique using an adaptive neuro-fuzzy," IEEE Commun. Lett., vol. 7, no. 2, pp. 55-57, Feb. 2003.

[44] K. C. Lee and P. Gardner, "Adaptive neuro-fuzzy inference system (ANFIS) digital predistorter for RF power amplifie linearization," IEEE Trans. Veh. Technol., vol. 55, no. 1, pp. 43-51, Jan. 2006.
[45] A. A. M. Saleh, "Frequency-independent and frequency-dependent nonlinear models of TWT amplifiers" IEEE Trans. Commun., vol. 29, no. 11, pp. 1715-1720, Nov. 1981.

[46] A. Saleh and J. Salz, "Adaptive linearization of power amplificatio in digital radio systems," Bell Syst. Tech. J., vol. 62, no. 4, pp. 1019-1033, Apr. 1983.

[47] C. Rapp, "Effects of HPA nonlinearity on 4-DPSK-OFDM-signal for a digital sound broadcasting system," in In Proc. 2nd Eur. Conf. Satellite Commun., Liege, Belgium, Oct. 1991, vol. 2, pp. 179-184.

[48] C. J. Clark, G. Chrisikos, M. S. Muha, A. A. Moulthrop, and C. P. Silva, "Time-domain measurement technique with application to wideband power amplifie modeling," IEEE Trans. Microw. Theory Techn., vol. 46, no. 12, pp. 2531-2540, Dec. 1998.

[49] H. Ku, M. D. Mckinley, and J. S. Kenney, "Extraction of accurate behavior models for power amplifier with memory effects using twotone measurements," in In Proc. IEEE MTT-S Int. Microw. Symp. Digest, Seatle, WA, Jun. 2002, vol. 1, pp. 139-142.

[50] J. Kim and K. Kanstantinou, "Digital predistortion of wideband signals based on power amplifie model with memory," IEE Electron. Lett., vol. 37, no. 23, pp. 1417-1418, Nov. 2001.

[51] M. Honkanen and S.-G. Haggman, "New aspects on nonlinear power amplifie modeling in radio communication system simulations," in In Proc. IEEE Int. Symp. On Personal, Indoor, and Mobile Comm (PIMRC), Helsinki, Finland, Sep. 1997, pp. 844-848.

[52] C.-S. Choi, Y. Shoji, H. Harada, R. Funada, S. Kato, K. Maruhashi, I. Toyoda, and K. Takahashi, "RF impairment models $60 \mathrm{GHz}$ band SYS/PHY simulation," Document IEEE 802.15-06-0477-01-003c, Nov. 2006.

[53] V. Erceg, M. Messe, A. Tarighat, M. Boers, J. Trachewsky, and C. Choi, “60 GHz impairments modeling," Document IEEE 802.11-09/1213r1., Nov. 2009.

[54] P. Jardin and G. Baudoin, "Filter look up table method for power amplifier linearization," IEEE Trans. Veh. Technol., vol. 56, no. 3, pp. 1076-1087, 2007.

[55] N. J. Gordon, D. J. Salmond, and A. F. M. Smith, "Novel approach to nonlinear/non-Gaussian Bayesian state estimation," Radar and Signal Process., IEE Proc. F, vol. 140, no. 2, pp. 107-113, Apr. 1993.

[56] D. Culibrk, O. Marques, D. Socek, H. Kalva, and B. Furht, "Neural network approach to background modeling for video object segmentation," IEEE Trans. Neural Netw., vol. 2, no. 5/6, pp. 673-689, Jun. 2005.

[57] T. Takagi and M. Sugeno, "Fuzzy identificatio of systems and its applications to modeling and control," IEEE Trans. Syst., Man, Cybern., vol. 15 , no. 1 , pp. 116-132, 1985.

[58] N. Bawane, A. G. Kothari, and D. P. Kothari, "ANFIS based HVDC control and fault identificatio of HVDC converter," HAIT J. Sci. Eng. $B$, vol. 18 , no. 6, pp. 1614-1627, Nov. 2007.

[59] J. S. R. Jang, "ANFIS: Adaptive-network-based fuzzy inference system," IEEE Trans. Syst., Man, Cybern., vol. 23, no. 3, pp. 665-685, Jun. 1993.

[60] Y. Li and P. Yang, "Data predistortion with adaptive fuzzy systems," in In Proc. IEE Int. Conf. Syst., Man, Cybern., 1999, vol. 6, pp. 168-172.

[61] L. Eng Aik and Y. S. O. Jayakumar, "A study of neuro-fuzzy system in approximation-based problems," Matematika, vol. 24, no. 2, pp. 113-130, 2008.

[62] SugenoT. Takagi, "Fuzzy identificatio of systems and its application to modeling and control," IEEE Trans. Syst., Man, Cybern., vol. 15, no. 1, pp. 116-132, 1985.

[63] M. D. McKinley, K. A. Remley, M. Myslinski, J. S. Kenney, D. Schreurs, and B. Nauwelaers, "EVM calculation for broadband modulated signals," in In Proc. 64th ARFTG Conf., Orlando, FL, Dec. 2004, pp. $45-52$. 